\title{
Acidentes de trabalho com enfermeiros de clínica médica envolvendo material biológico
}

\author{
Accidents with biological material involving clinical medicine nurses \\ Accidentes de trabajo con enfermeros de clínica médica involucrando material biológico
}

\author{
Larissa Pereira Costa'; Paula Raquel dos Santos ${ }^{\text {II }}$; Alessandra da Terra Lapa ${ }^{I I I}$; Thelma SpindolaIV
}

\begin{abstract}
RESUMO: Estudo descritivo e quantitativo que teve o objetivo de verificar se os acidentes com material biológico em enfermeiros de clínica médica ocorrem com maior frequência entre os residentes de enfermagem. O estudo foi realizado em um hospital universitário da cidade do Rio de Janeiro, em 2013. Os dados foram coletados com emprego da técnica de análise documental, com auxílio de um roteiro estruturado. As informações foram captadas nas fichas de notificação de acidentes com material biológico, no período de 2007 a 2011, envolvendo os profissionais de enfermagem da instituição. Os resultados evidenciam que, no período analisado, ocorreram 260 acidentes com profissionais da instituição. Dos 27 (100\%) acidentes que envolveram enfermeiros de clínica médica, $21(77,7 \%)$ ocorreram com residentes. Pode-se concluir que, no conjunto amostral analisado, os enfermeiros residentes da clínica médica estão envolvidos na maioria dos acidentes registrados.
\end{abstract}

Palavras-Chave: Enfermagem; exposição a agentes biológicos; enfermagem do trabalho; enfermagem clínica.

\begin{abstract}
In order to establish whether accidents with biological material among clinical nurses occur more frequently among nurse residents, a descriptive, quantitative study was conducted at a university hospital in Rio de Janeiro city in 2013. Data were collected by document analysis technique following a structured guide. Information was drawn from forms completed between 2007 and 2011 reporting accidents with biological material involving the institution's nursing personnel. The results show that in the study period there were 260 accidents involving staff at the institution. Of the $27(100 \%)$ accidents involving clinical medicine nurses, $21(77.7 \%)$ involved nurse residents. From the sample analyzed, it can be concluded that clinical medicine nurse residents are involved in most of the accidents recorded.

Keywords: Nursing; exposure to biological agents; nursing work; clinical nursing.
\end{abstract}

RESUMEN: Estudio descriptivo, cuantitativo que tuvo como objeto verificar si los accidentes con material biológico en enfermeros de clínica médica ocurren con más frecuencia entre los residentes de enfermería. Tuvo lugar en un hospital universitario de la ciudad de Rio de Janeiro, en 2013. Los datos fueron recolectados haciendo uso de la técnica de análisis de documentos, con el auxilio de un guión estructurado. Las informaciones han sido captadas en los registros de notificación de accidentes con material biológico, entre 2007 y 2011, involucrando a los profesionales de enfermería de la institución. Los resultados muestran que, en el período analizado, han ocurrido 260 accidentes con profesionales de la institución. De los $27(100 \%)$ accidentes que han involucrado a enfermeros de clínica médica, $21(77,7 \%)$ ocurrieron con residentes. Se puede concluir que, en el conjunto de muestras analizado, los enfermeros residentes de la clínica médica están involucrados en la mayoría de los accidentes registrados.

Palabras Clave: Enfermería; exposición a agentes biológicos; enfermería del trabajo; enfermería clínica.

\section{INTRODUÇÃO}

Este estudo tem como objeto a ocorrência de acidentes com material biológico envolvendo enfermeiros residentes de clínica médica.

Os trabalhadores da área da saúde estão expostos a situações que podem causar infecções transmitidas por microrganismos presentes no sangue e fluidos orgânicos. Esse problema acentua-se em hospitais, nos quais há um grande número de trabalhadores de enfermagem. Dessa forma, medidas de biossegurança devem ser adotadas pelas instituições e pelos trabalhadores para minimizar tal exposição ${ }^{1}$.

A vivência como profissionais de enfermagem e a prestação de assistência direta aos clientes suscitaram a escolha deste objeto considerando que os profissionais

'Enfermeira. Mestranda em Enfermagem da Faculdade de Enfermagem da Universidade do Estado do Rio de Janeiro. Residência em Enfermagem em Clínica Médica pela Universidade do Estado do Rio de Janeiro. Brasil. E-mail: larissac bj@yahoo.com.br.

"Enfermeira. Doutora em Enfermagem. Professora Adjunta do Departamento de Enfermagem de Saúde Pública. Faculdade de Enfermagem da Universidade do Estado do Rio de Janeiro. Brasil. E-mail: paularaquel.enf@gmail.com.

IIIEnfermeira. Mestranda em Enfermagem da Faculdade de Enfermagem da Universidade do Estado do Rio de Janeiro. Professora Substituta do Departamento de Enfermagem de Saúde Pública. Faculdade de Enfermagem da Universidade do Estado do Rio de Janeiro. Brasil. E-mail: aless.lapa@yahoo.com.br.

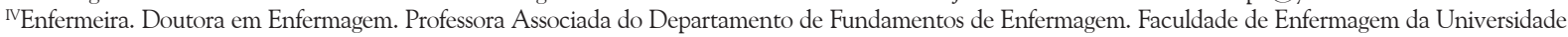
do Estado do Rio de Janeiro. Brasil. E-mail: tspindola.uerj@gmail.com.

varte integrante do relatório de pesquisa intitulado Acidentes de trabalho com enfermeiros envolvendo material biológico em clínica médica, aprovado no Programa de Pós-Graduação - modalidade Residência em Enfermagem em Clínica Médica, da Universidade do Estado do Rio de Janeiro, 2013. 
enfermeiros, por desenvolverem atividades junto à clientela, estão em eminente risco de se acidentarem com materiais biológicos.

$\mathrm{Na}$ atuação em hospital universitário, pode-se observar que, no grupo dos enfermeiros, os residentes de enfermagem estão muito expostos aos riscos ocupacionais em função das condições estruturais do ambiente de trabalho e da falta de medidas de biossegurança adequadas. Além disso, a elevada carga horária semanal de trabalho contribui para o cansaço físico e mental, levando a um menor rendimento e atenção ao trabalho, o que está intimamente ligado à ocorrência de acidentes.

O ambiente de trabalho nem sempre oferece materiais adequados e em quantidade necessária para a execução dos serviços, como, por exemplo, luvas, aventais, máscaras e óculos. Além da escassez de material, há problemas como ausência de iluminação adequada do ambiente, de pias para lavagem das mãos, de dispenser com álcool em gel para higienização das mãos e de recipiente apropriado para descarte de material perfurocortante, sendo estas estruturas fundamentais para viabilizar a aplicação dos métodos de biossegurança pelos trabalhadores.

A principal via de transmissão de microrganismos durante a assistência prestada aos pacientes são as mãos do profissional, pois a pele é um possível reservatório de diversos microrganismos, que podem se transferir por meio de contato direto ou indireto, pelo contato com objetos e superfícies contaminados ${ }^{2}$.

Quando se fala em biossegurança, no risco de se adquirir infecção ou na possibilidade da contaminação, logo se pensa em maneiras de evitar e/ou minimizar estes fatores de risco.

A higienização correta das mãos é o principal método para prevenir e controlar infecções no ambiente hospitalar, sendo também o mais importante e menos dispendioso. No entanto, muitos profissionais de saúde o fazem de maneira não satisfatória, sem observar as recomendações, não realizando a higienização das mãos em aproximadamente $60 \%$ das vezes que seria necessário ou indicado. Esta medida, portanto ainda tem sido um grande desafio devido à baixa adesão dos profissionais da área da saúde à lavagem das mãos ${ }^{3}$.

De acordo com a Norma Regulamentadora 32 (NR-32), que dispõe sobre a segurança e saúde dos trabalhadores dos serviços de saúde, o uso de luvas não substitui o processo de lavagem das mãos, o que deve ocorrer, no mínimo, antes e depois de seu uso ${ }^{4}$.

Os equipamentos de proteção individual (EPI) possuem a função de reduzir danos e agravos nos casos de acidentes e são adotados como precauçõespadrão, sendo o seu uso indispensável no atendimento aos pacientes. No entanto, muitos acidentes ainda acontecem nos serviços de saúde, sendo imprescindível a notificação destes e o acompanhamento dos profissionais.

Os acidentes do trabalho com exposição a material biológico potencialmente contaminado são considerados, no Brasil, um agravo de notificação compulsória e deve ser notificado em ficha padronizada pelo Ministério da Saúde no Sistema de Informação de Agravos de Notificação (SINAN) e em redes sentinelas, como os Centros de Referência em Saúde do Trabalhador (CEREST) ${ }^{5}$.

A partir desta contextualização, foi selecionado como problema para a investigação: Qual a ocorrência de acidentes de trabalho com enfermeiros residentes de clínica médica, envolvendo material biológico? Foi determinado como objetivo verificar se os acidentes com material biológico em enfermeiros de clínica médica ocorrem com maior frequência entre os residentes de enfermagem.

Espera-se que os resultados do estudo possam fomentar o interesse dos profissionais de saúde na utilização e uso correto dos equipamentos de proteção individual, com a finalidade de minimizar a ocorrência de acidentes de trabalho envolvendo material biológico.

\section{Revisão de Literatura}

A rotina de trabalho em estabelecimentos de saúde exige que o trabalhador esteja atento às normas de biossegurança para garantir a proteção da sua saúde. Biossegurança é concebida como um conjunto de medidas voltadas para a prevenção, a redução ou a eliminação de riscos inerentes às atividades de pesquisa, produção, ensino, desenvolvimento tecnológico e prestação de serviços ${ }^{4}$.

A exposição ocupacional a material biológico provoca danos à integridade física, mental e social constituindo os acidentes, frequentes entre os trabalhadores de enfermagem. Alguns fatores são predisponentes para estes agravos como o número insuficiente de trabalhadores, a sobrecarga de trabalho, jornadas fatigantes, continuidade da assistência em turnos e plantões noturnos, desgaste físico e emocional, capacitação técnica deficiente, falta de atenção, excesso de confiança, utilização de materiais inadequados, estresse e a não adoção das medidas de precauçõespadrão ${ }^{6}$.

Essa exposição deve ser avaliada quanto ao potencial de transmissão do Vírus da Imunodeficiência Adquirida (HIV) e do Vírus da Hepatite B (HBV) e da Hepatite C (HCV), quanto ao tipo de exposição, tipo e quantidade de fluido e tecido, status sorológico da fonte, status sorológico do acidentado e suscetibilidade do profissional exposto ${ }^{1}$. 
No Brasil, os acidentes do trabalho, o adoecimento e o absenteísmo entre profissionais da área da saúde despertaram a atenção do Ministério do Trabalho e Emprego que, atendendo às solicitações das entidades que representam as diversas categorias de trabalhadores da saúde, inseriu na legislação, em 2005, a NR 32 - Segurança e Saúde no Trabalho nos Estabelecimentos de Saúde. Esta é considerada de extrema importância, pois aborda questões de segurança e saúde no trabalho, no setor da saúde?

Tal norma regulamentadora preconiza que as instituições de saúde deverão implantar ações de promoção, proteção e recuperação da saúde dos trabalhadores que atuam na prestação de assistência à saúde. A NR-32 possui três grandes eixos: a capacitação contínua dos trabalhadores; os programas que tratam dos riscos; e as medidas de proteção contra os riscos?

\section{Metodologia}

Estudo descritivo, quantitativo, com emprego da técnica de análise documental.

Foi realizado em um hospital universitário, na cidade do Rio de Janeiro, em 2013. Os dados foram coletados com emprego da técnica de análise documental, com auxílio de um roteiro estruturado. As informações foram captadas nas fichas de notificação de acidentes com material biológico, no período de 2007 a 2011, envolvendo os profissionais de enfermagem da instituição. As fichas encontram-se disponíveis em uma base de dados digital, no setor responsável pela notificação e atendimento aos profissionais acidentados. Elas contêm informações como a data do acidente, a idade, o sexo, a ocupação do acidentado, o tipo de acidente, o objeto causador, o material biológico envolvido, entre outras informações.

A equipe de enfermagem desse hospital de ensino é composta por enfermeiros efetivos, enfermeiros em Treinamento Profissional Bolsista (TPB), técnicos de enfermagem efetivos, técnicos de enfermagem contratados, auxiliares de enfermagem, residentes de enfermagem e, nas aulas práticas, professores de enfermagem e internos de enfermagem.

O hospital, sede da pesquisa, é composto por 303 enfermeiros efetivos, 278 enfermeiros TPB, 934 técnicos de enfermagem efetivos, 435 técnicos de enfermagem contratados. Ao todo são 581 enfermeiros e 1369 técnicos de enfermagem. Desse total, 40 enfermeiros e 84 técnicos de enfermagem são de clínica médica. Outra categoria agrega os residentes de enfermagem. Ao todo são 141 residentes de enfermagem, sendo 73 residentes de enfermagem do primeiro ano e 68 residentes de enfermagem do segundo ano. Entre estes, 23 são residentes de enfermagem de clínica médica, sendo 12 residentes de enfermagem do primeiro ano e 11 residentes de enfermagem do segundo ano.
Para o levantamento dos dados, foi utilizado um roteiro estruturado com nove perguntas fechadas, construídas com base na literatura, na ficha de notificação de acidentes e doenças do trabalho da instituição da pesquisa e na ficha de notificação de acidentes de trabalho com exposição a material biológico, construído pelo SINAN - Ministério da Saúde.

Os critérios de inclusão foram: ser enfermeiro e/ ou residente de enfermagem; ter sofrido algum acidente com material biológico em uma unidade de clínica médica; estar dentro do recorte temporal de cinco anos. Os critérios de exclusão foram: não ser enfermeiro e/ ou residente de enfermagem; não ter sofrido nenhum acidente por material biológico; acidentes que ocorreram há mais de cinco anos; de 2007 a 2011 acidentes que não tenham ocorrido em clínica médica.

O estudo foi aprovado pelo Comitê de Ética em Pesquisa da instituição, conforme a Resolução ${ }^{\circ}$ 466/12 do Conselho Nacional de Saúde, obtendo a numeração 285.692.

Por se tratar de uma pesquisa que envolve seres humanos de forma indireta, já que os dados foram obtidos pelo manejo das fichas de notificação de acidentes dos profissionais, pela quantidade de sujeitos que foram envolvidos e por considerar que alguns deles poderiam não ter mais vínculo empregatício com o hospital, sede da pesquisa, foi solicitado ao Comitê de Ética em Pesquisa a autorização para coleta de dados sem a necessidade de obtenção do Termo de Consentimento Livre e Esclarecido (TCLE).

Os dados expostos foram coletados em dois meses, após a aprovação do Comitê de Ética, no setor de notificação e atendimento aos profissionais acidentados desse hospital.

As informações obtidas foram armazenadas em uma planilha do software Microsoft Office Excel 2007 e analisadas com suporte da estatística descritiva.

\section{Resultados e Discussão}

Os dados evidenciam que a ocorrência de acidentes com exposição a material biológico ainda é elevada no ambiente hospitalar e, consequentemente, com a equipe de enfermagem.

No recorte temporal investigado ocorreram 260 acidentes com a equipe de enfermagem, sendo $27(10,3 \%)$ com enfermeiros na clínica médica. Ver Tabela 1.

Os acidentes envolvendo os enfermeiros apresentaram a seguinte caracterização: sete enfermeiros de clínica médica acidentaram-se em 2007. Entre os sete, cinco eram enfermeiros residentes. Em 2008, também foram sete enfermeiros acidentados, sendo seis enfermeiros residentes em clínica médica. No ano de 2009, foram notificados cinco acidentes, en- 
TABELA 1: Perfil geral dos acidentes. Rio de Janeiro, RJ, 2013.

\begin{tabular}{lcccccc}
\hline \multicolumn{1}{c}{ Perfil geral dos acidentes } & $\mathbf{2 0 0 7}$ & $\mathbf{2 0 0 8}$ & $\mathbf{2 0 0 9}$ & $\mathbf{2 0 1 0}$ & $\mathbf{2 0 1 1}$ & Total \\
\hline Acidentes envolvendo equipe de enfermagem & 65 & 23 & 81 & 71 & 20 & 260 \\
Acidentes envolvendo equipe de enfermagem de Clínica Médica & 14 & 7 & 14 & 10 & 4 & 49 \\
Acidentes envolvendo enfermeiros de Clínica Médica & 7 & 7 & 5 & 6 & 2 & 27 \\
Acidentes envolvendo enfermeiros residentes de Clínica Médica & 5 & 6 & 5 & 5 & 0 & 21 \\
\hline Total & $\mathbf{1 4 8}$ & $\mathbf{1 4 5}$ & $\mathbf{1 6 6}$ & $\mathbf{1 6 9}$ & $\mathbf{4 7}$ & $\mathbf{6 7 5}$ \\
\hline
\end{tabular}

volvendo enfermeiros de clínica médica e todos eram residentes. Em 2010, foram seis acidentes notificados com enfermeiros, sendo cinco ocorridos entre os residentes de clínica médica. Em 2011, dois acidentes com enfermeiros foram notificados, entretanto não abrangeram residentes de enfermagem.

Entre os 27 (100\%) acidentes envolvendo enfermeiros, na clínica médica, os enfermeiros residentes estiveram envolvidos na maioria deles - $21(77,7 \%)$ - e com exposição a material biológico, no período estudado.

Ressalta-se que a falta de experiência e ansiedade pode contribuir para a ocorrência de acidentes. Estar constantemente em situações de aprendizado, supervisão e avaliação favorece o aumento da ansiedade e estresse ${ }^{8}$.

Com relação à faixa etária dos profissionais envolvidos em acidentes com material biológico, cinco acidentes do ano de 2007 aconteceram com profissionais de 21 a 30 anos, num total de sete. Todos os sete registros do ano de 2008 e os cinco registros de 2009 foram realizados com profissionais de 21 a 30 anos. $O$ ano de 2010 teve duas notificações com profissionais de até 20 anos e três notificações com enfermeiros de 21 a 30 anos. E, em 2011, as duas notificações envolviam profissionais de 21 a 30 anos.

A grande participação de jovens profissionais na ocorrência de acidentes com materiais biológicos pode explicar o número elevado de acidentes entre enfermeiros residentes. Este índice pode estar associado ao tempo reduzido de exercício da profissão.

Jovens profissionais possuem uma grande expectativa no começo da carreira e, quando ingressam no universo do trabalho, percebem as dificuldades da realidade que é encontrada. Essa situação tende a levar este jovem ao estresse e a enfermidades no trabalho, inclusive acidentes?.

Considerando o sexo, verificou-se que, no ano de 2007 e 2010, nenhum dos acidentes notificados envolveu profissionais do sexo masculino. Já no ano de 2008, cinco profissionais eram do sexo feminino num total de sete e em 2009, quatro eram do sexo feminino num total de cinco. No ano de 2011, foram registrados apenas dois acidentes com enfermeiros, sendo um do sexo masculino e um do sexo feminino.

Essa maior ocorrência dos aciden $\neg$ tes entre profissionais do sexo feminino pode ser justificada considerando que a enfermagem é uma categoria majoritariamente feminina e com expressiva representatividade nos serviços de saúde ${ }^{10}$.

Outra justificativa é a de que o número de mulheres que ingressam nos cursos de nível superior é elevado. O Censo da Educação Superior de 2013 indica o crescimento do número de mulheres que ingressam, se matriculam e concluem a graduação, principalmente nos cursos da área da saúde ${ }^{11}$.

Classificados de acordo com o tipo da exposição, em 2007 e 2008 ocorreram cinco acidentes percutâneos notificados entre os sete totais. Em 2009, três acidentes percutâneos foram registrados no total de cinco. No ano de 2010, foram notificados quatro acidentes percutâneos entre os seis e, em 2011, houve notificação de um acidente percutâneo e de um acidente com mucosa oral/ocular.

As agulhas são os objetos causadores do maior número de acidentes, entretanto boa parte dos acidentes registrados poderia ter sido evitada pela adoção de medidas de precaução-padrão, tais como a não recapagem de agulhas e o descarte adequado dos perfurocortantes ${ }^{12}$.

Nos anos de 2007, 2009 e 2010, todos os casos notificados de acidentes tiveram a agulha como objeto envolvido no acidente. Em 2007, foram sete casos, em 2009 foram cinco e em 2010 foram seis casos. No ano de 2008, dos sete acidentes, seis foram com agulha. Apenas um acidente envolvia lâmina/lanceta. Em 2011, a notificação de objeto causador do acidente foi ignorada nos dois casos registrados.

O sangue é o material orgânico mais envolvido nos acidentes com exposição a materiais biológicos ${ }^{13}$.

Em 2007, das sete notificações todas envolviam sangue e, em 2009, foram todos os cinco registros envolvidos com sangue. No ano de 2008, foram notificados seis acidentes envolvendo sangue e um acidente envolvendo fluido com sangue. Em 2010, dos seis registros de acidentes, quatro envolviam sangue e dois não informaram o material orgânico envolvido. Em 2011, as duas notificações ocorridas, também não informaram o material orgânico envolvido.

O uso de luvas é uma prática recomendada internacionalmente, sendo considerada uma importante medida preventiva de exposição a material biológico, pois minimiza o risco de contato com sangue e outros fluidos corpóreos, capazes de transmitir micro-organismos patogênicos, a exemplo dos agentes HCV, HBV e HIV ${ }^{13}$. 
Em acidentes com perfurocortantes, o uso de luvas pode reduzir o volume de sangue injetado por agulhas de sutura em $70 \%$ e por agulhas para o tratamento intravenoso de $35 \%$ a $50 \%$, considerando que parte desse fluido pode permanecer no bisel e/ou no interior da agulha. Assim, apesar de não impedir a perfuração, as luvas funcionam como uma barreira mecânica auxiliar para diminuir o risco de contaminação em casos de acidentes ${ }^{14}$.

Com base na Ficha de Notificação de Acidentes e Doenças do Trabalho da instituição da pesquisa, as circunstâncias dos acidentes compreendem três momentos: durante o procedimento; depois do procedimento e antes do descarte; e durante ou após o descarte.

Nos anos de 2007 e 2008, foram notificados cinco acidentes que ocorreram durante o procedimento e dois que ocorreram depois do procedimento e antes do descarte do material utilizado. Em 2009, foram registrados quatro acidentes durante o procedimento e apenas um depois do procedimento e antes do descarte. Foram notificados, em 2010, cinco acidentes durante o procedimento e um depois do procedimento e antes do descarte. Já no ano de 2011, um acidente foi notificado durante o procedimento e um depois do procedimento e antes do descarte. Ver Tabela 2.

A maioria dos acidentes ocorreu no momento em que o profissional estava realizando o procedimento, o que reforça a importância do uso de luvas durante essas situações, conforme evidencia a Tabela 2.

O perfil dos enfermeiros de clínica médica, envolvidos em acidentes com exposição a material biológico, mostra-se semelhante. São enfermeiros com a mesma faixa etária (de 21 a 30 anos), sexo (feminino), tipologia de acidente - percutâneo-, com o mesmo objeto causador (agulha) e contato com o mesmo material orgânico - sangue. Observa-se, também, que os acidentes, em geral, acontecem sob a mesma circunstância, ou seja, durante o procedimento que está sendo realizado.
O uso de equipamentos de proteção individual foi registrado em desuso pelo profissional no momento do acidente, na maioria dos casos.

No ano de 2007, dos sete enfermeiros acidentados, quatro não usavam equipamento de proteção individual (EPI) no momento do acidente. Em 2008, também, foram sete enfermeiros acidentados, porém seis não usavam EPI. Dos cinco acidentados em 2009, quatro não usavam o EPI. Em 2010, quatro profissionais não usavam EPI no momento do acidente e dois registros não informaram a respeito, num total de seis acidentes. Em 2011, nos dois acidentes notificados, não fizeram uso de EPI. Ver Tabela 3.

No levantamento de qual EPI foi mais usado pelos profissionais acidentados, entre os que estavam munidos do equipamento no momento do acidente, a luva aparece com um registro e a máscara com dois, no ano de 2007. Em 2008, apenas um acidente com uso de luva foi notificado. Em 2009, há um registro do uso de avental e, nos anos de 2010 e 2011, não houve nenhuma notificação com relação ao uso de EPI.

O uso regular do EPI é extremamente relevante para prevenir a exposição aos materiais biológicos, oferecendo maior segurança para profissionais e pacientes. Para que esta segurança seja efetiva, é preciso que, além da adesão, estes equipamentos sejam utilizados de maneira correta, e que sempre sejam verificados se estão limpos e conservados ${ }^{15,16}$.

Várias circunstâncias predispõem a ocorrência dos acidentes de trabalho com material biológico. Há os condicionantes individuais, como a não adesão ao uso dos EPI, o reencape das agulhas contaminadas, o descarte inadequado do material contaminado, assim como a dupla jornada de trabalho que leva à fadiga, ao cansaço e à falta de atenção ${ }^{10}$. Há, ainda, os condicionantes institucionais, como a falta de capacitação e treinamento oferecidos por parte dos gestores aos profissionais, a agitação do serviço, o estresse, a falta de recursos humanos, a inadequação de recipientes para descarte dos materiais contaminados, entre outros ${ }^{10,17}$.

TABELA 2: Circunstância do acidente. Rio de Janeiro, RJ, 2013.

\begin{tabular}{lccccc}
\hline \multicolumn{1}{c}{ Circunstância do acidente } & 2007 & 2008 & 2009 & 2010 & 2011 \\
\hline Durante o procedimento & 5 & 5 & 4 & 5 & 1 \\
Depois do procedimento e antes do descarte & 2 & 2 & 1 & 1 & 1 \\
Durante ou após o descarte & - & - & - & - & - \\
\hline Total & 7 & 7 & 5 & 6 & 2 \\
\hline
\end{tabular}

TABELA 3: Uso de equipamento de proteção individual. Rio de Janeiro, RJ, 2013.

\begin{tabular}{lccccc}
\hline Uso de equipamento de proteção individual & 2007 & $\mathbf{2 0 0 8}$ & $\mathbf{2 0 0 9}$ & $\mathbf{2 0 1 0}$ & $\mathbf{2 0 1 1}$ \\
\hline Usa EPI & 3 & 1 & 1 & 0 & 0 \\
Não usa EPI & 4 & 6 & 4 & 4 & 2 \\
Ignorado & 0 & 0 & 0 & 2 & 0 \\
\hline Total & 7 & 7 & 5 & 6 & 2 \\
\hline
\end{tabular}


Nesse contexto, torna-se relevante que os gestores incentivem o uso dos EPI entre os profissionais de saúde durante os procedimentos. De forma análoga, devem conscientizá-los da necessidade de adequação às normas de reuso de artigos, para reduzir o grau de exposição, sobretudo durante reprocessamentos não recomendados. $O$ trabalho de orientação sobre descarte e manuseio do lixo biológico, também, deve ser reforçado entre os profissionais ${ }^{10,12-16}$.

\section{ConClusão}

Os achados evidenciam a ocorrência de acidentes com material biológico entre os profissionais de enfermagem, principalmente com os enfermeiros residentes, por inúmeros fatores como: a baixa experiência, a constante situação de cobrança e avaliação como aluno/profissional em fase de aprimoramento, as condições de trabalho, a baixa adesão ao uso de EPI, a excessiva jornada de trabalho, o estresse, entre outros.

Os residentes de enfermagem, apesar de serem profissionais registrados no Conselho regulamentador da profissão, ainda estão em processo de aprendizagem, de aprimoramento prático-teórico e necessitam, portanto, de preceptores/supervisores em suas atividades diárias. Além disso, precisam do ensino acerca das condutas práticas e da valorização do uso de EPI, considerando os riscos a que ficam expostos no ambiente laboral.

A educação continuada é um recurso importante que deve ser adotada nas instituições de saúde para reciclagem dos profissionais e equipes de saúde, para aprendizagem, e como estratégia para estimular a adoção de medidas preventivas para reduzir o número de acidentes com materiais biológicos entre os trabalhadores.

Os objetivos deste estudo foram alcançados, tendo-se evidenciado que a maioria dos acidentes com material biológico na clínica médica, no período analisado, teve o envolvimento de enfermeiros residentes.

Não houve dificuldades para o acesso às informações descritas nas fichas de notificação da instituição sede da pesquisa, considerando que os dados se encontram em uma base digital organizada, o que facilitou a captação.

As limitações do estudo foram a ausência de algumas informações nas fichas de notificação de acidente da instituição, a rotatividade de residentes por ano e a alta carga horária do programa de residência, o que dificultou a busca pelas informações e a sua análise mais aprofundada.

Esta investigação traz contribuições para a instituição sede da pesquisa que pode promover ações com propósito de reduzir o número de acidentes ocupacionais entre os trabalhadores de enfermagem e fiscalizar os casos de subnotificação. Espera-se que esse tipo de acidente no ambiente hospitalar seja cada vez mais reduzido, com oferta de melhores condições de trabalho e qualidade de vida para os profissionais. Destaca-se que este assunto não se esgota com esta pesquisa. Novos estudos devem ser realizados para ampliar a discussão e a produção científica acerca do tema.

\section{REFERÊNCIAS}

1.Soares LG, Labronici LM, Maftum MA, Sarquis LMM, Kirchhof AL. Risco biológico em trabalhadores de enfermagem: promovendo a reflexão e a prevenção. Cogitare Enferm. 2011; 16: 261-7.

2.Oliveira KB. Importância da higiene das mãos no controle e prevenção da infecção hospitalar. Universo da Enfermagem. 2014; 3: 36-42.

3.Lorenzini E, Costa TC, Silva EF. Prevenção e controle de infecção em unidade de terapia intensiva neonatal. Rev Gaúcha Enferm. 2013; 34: 107-13.

4.Brand CI, Fontana RT. Biossegurança na perspectiva da equipe de enfermagem de unidades de tratamento intensivo. Rev Bras Enferm. 2014; 67: 78-84.

5.Valim MD, Marziale MHP, Hayashida M, RichartMartínez M. Ocorrência de acidentes de trabalho com material biológico potencialmente contaminado em enfermeiros. Acta Paul Enferm. 2014; 27: 280-6.

6.Soares LG, Sarquis LMM, Kirchhof ALC, Felli VEA. Multicausalidade nos acidentes de trabalho da enfermagem com material biológico. Rev Bras Enferm. 2013; 66: 854-9.

7.Marziale MHP, Galon T, Cassiolato FL, Girão FB. Implantação da Norma Regulamentadora 32 e o controle dos acidentes de trabalho. Acta Paul Enferm. 2012; 25: 859-66.

8.Canalli RTC, Moriya TM, Hayashida M. Acidentes com material biológico entre estudantes de enfermagem. Rev enferm UERJ. 2010; 18: 259-64.

9.Trindade LL, Grisa CC, Ostrovski VP, Adamy EK, Ferraz L, Amestoy SC et al. Absentismo en el equipo de enfermería en el ambiente hospitalario. Enferm glob [online]. 2014; 13: 138-46.

10.Vieira M, Padilha MI, Pinheiro RDC. Análise dos acidentes com material biológico em trabalhadores da saúde. Rev Latino-Am Enfermagem. 2011; 19: 1-8.

11.Ministério da Educação (Br). Instituto Nacional de Estudos e Pesquisas Educacionais Anísio Teixeira. Censo da Educação Superior 2013. Brasília (DF): Ministério da Educação; 2013.

12.Lima LM, Oliveira CC, Rodrigues KMR. Exposição ocupacional por material biológico no Hospital Santa Casa de Pelotas - 2004 a 2008. Esc Anna Nery. 2011; 15: 96-102.

13.Marziale MH, Zapparoli AS, Felli VE, Anabuki MH. Rede de prevenção de acidentes de trabalho: uma estratégia de ensino a distância. Rev Bras Enferm. 2010; 63: 250-6.

14.Ribeiro PC, Ribeiro ACC, Lima Júnior FPB. Perfil dos acidentes de trabalho em um hospital de Teresina, PI. Cogitare Enferm. 2010; 15: 110-6. 
15.Lapa AT, Silva JM, Spindola T. A ocorrência de acidentes por material perfurocortante entre trabalhadores de enfermagem intensivista. Rev enferm UERJ. 2012; 20: 642-7.

16.Guilarde AO, Oliveira AM, Tassara M, Oliveira B, Andrade SS. Acidentes com material biológico entre profissionais de hospital universitário em Goiânia. Revista de Patologia Tropical. 2010; 39: 131-6.

17.Kestemberg CCF, Felipe ICV, Rossone FO, Delphim LM, Teotonio MC. $\mathrm{O}$ estresse do trabalhador de enfermagem: estudo em diferentes unidades de um hospital universitário. Rev enferm UERJ. 2015; 23: 45-51. 